\title{
Second-harmonic generation by resonance absorption on nanoplasmas in the bulk of dielectrics
}

\author{
Kazem Ardaneh, Mostafa Hassan, Remi Meyer, Remo Giust, Francois Courvoisier \\ FEMTO-ST Institute, Univ. Bourgogne Franche-Comté, UMR CNRS 6174, \\ $15 B$ avenue des Montboucons, 25030 Besançon, France
}

Second-harmonic (SH) radiation spectrum is an important diagnostic in nonlinear laser-plasma interactions. SH emission pattern provides useful information about the absorption mechanisms in the plasma. To date, experimental and theoretical works related to $\mathrm{SH}$ emission in plasmas are limited to plasmas at the surface of solids. Here, for the first time to our knowledge, we have experimentally characterized SH for plasmas inside the bulk of dielectrics with inversion symmetry. This is performed using single-shot femtosecond Bessel pulses in fused silica, glass and sapphire. This configuration creates dense nano-plasmas (diam. $\approx 200$ to $500 \mathrm{~nm}$ ) which relaxation in the material is capable of creating Warm Dense Matter [1] and lately opening voids [2].

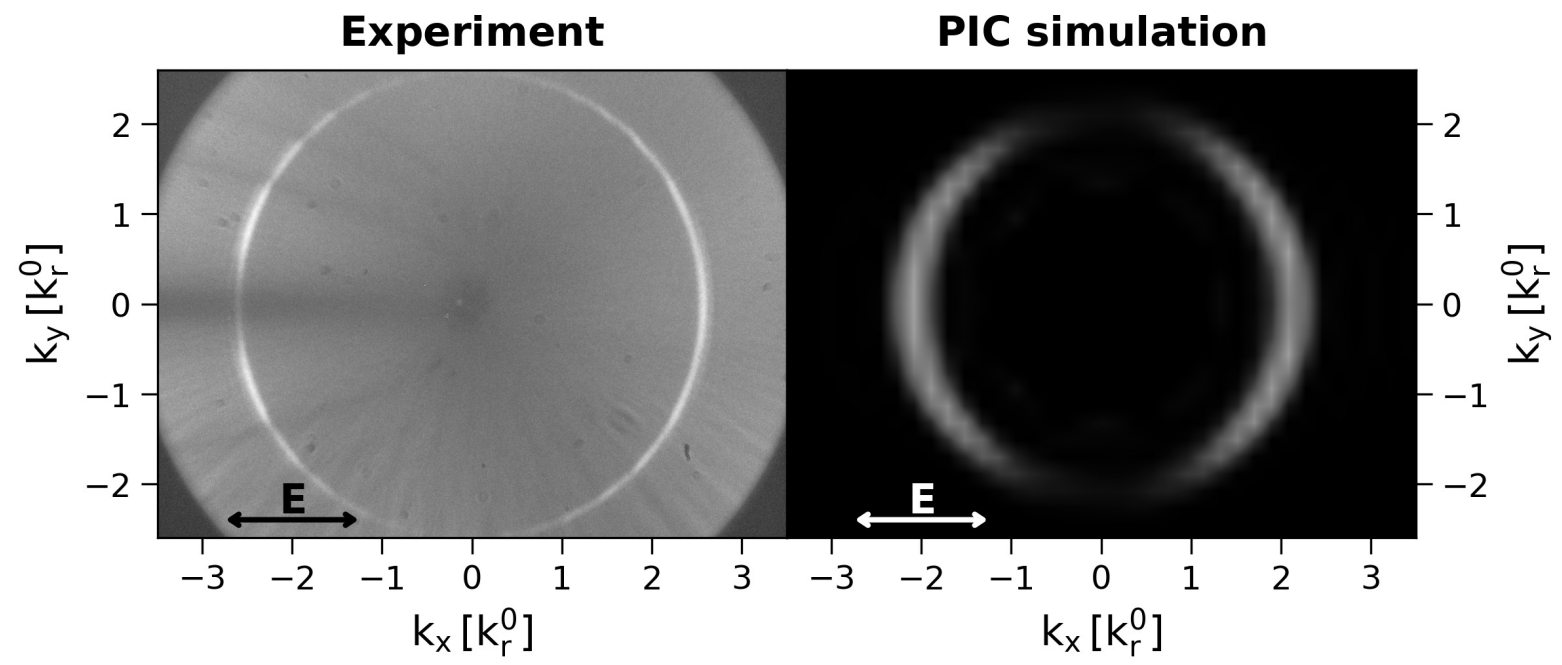

Figure 1: SH spatial radiation spectrum from experiment (left), and from PIC simulation (right) in sapphire. The axes are normalized in terms of the Bessel beam transverse spatial frequency $k_{r}^{0}$.

In our experiments, a single $100 \mathrm{fs}$ Bessel pulse at central wavelength $800 \mathrm{~nm}$ was focused in the bulk of transparent dielectrics at intensities of $10^{14} \mathrm{Wcm}^{-2}$, in conditions where voids can be opened [1,2]. Second harmonic emission was recorded in a single-shot mode in the far-field. Figure 1 shows the spatial spectrum of SH for the experiments and Particle-In-Cell (PIC) simulations. The simulations have been performed using EPOCH PIC code [3]. We have observed narrow emission lobes parallel to the laser polarization at $k_{r} \sim 2 k_{r}^{0}$. A background emission is also present in the experiments that originates from the blackbody emission of the plasma at $\sim \mathrm{ns}$ timescales after the pulse. The analysis of $\mathrm{SH}$ radiation reveals that the resonance absorption mechanism occurs on an overcritical nanoscale plasma. An excellent agreement is found between experiments and simulations for several diagnostics in addition to the second harmonic pattern.

In conclusion, we have observed for the first time second harmonic emission from over-dense plasmas in the bulk of materials in the single-shot configuration. The intense laser-plasma interaction has been investigated using PIC simulations. These reveal the microphysics at play and the role of electron plasma waves. We anticipate that this approach will find new applications in laser-plasma diagnostics and nonlinear optics of very intense fields.

This research has received funding from H2020 European Research Council (ERC) under grant agreement 682032-PULSAR. This work was granted access to HPC resources PRACE (PULSARPIC PRA19_4980), TGCC (A0070511001), and Mésocentre de Calcul de Franche-Comté.

References

[1] J. D. Hoyo, R. Meyer, L. Furfaro, and F. Courvoisier. "Nanoscale confinement of energy deposition in glass by double ultrafast Bessel pulses". Nanophotonics, 1(ahead-of-print), (2020).

[2] L. Rapp, R. Meyer, R. Giust, et al, "High aspect ratio micro-explosions in the bulk of sapphire generated by femtosecond Bessel beams," Sci. Rep 6, 34286 (2016).

[3] T. D. Arber, K. Bennett, C. S. Brady, et al, "Contemporary particle-in-cell approach to laser-plasma modelling," Plasma Phys. Control. Fusion 57, 113001 (2015). 